\title{
AN APPLICATION OF THE LEMMA ON THE LOGARITHMIC DERIVATIVE IN SEVERAL COMPLEX VARIABLES DUE TO A. VITTER
}

\author{
Dedicated to Professor Mitsuru Ozawa on his 60-th birthday
}

\section{BY SEIKI MORI}

1. A. Vitter [6] generalized Nevanlinna's lemma on logarithmic derivative to the case of meromorphic functions $f: \boldsymbol{C}^{n} \rightarrow \boldsymbol{P}^{1}(\boldsymbol{C}) \cong \boldsymbol{C} \cup\{\infty\}$, and he obtained the defect relation for holomorphic (or meromorphic) mappings $f: \boldsymbol{C}^{n} \rightarrow \boldsymbol{P}^{m}(\boldsymbol{C})$ and a collectionn of hyperplanes in general position by using the method due to H. Cartan [2]. Furthermore, he obtained the following generalization of a result of H. Milloux (see Hayman [4, p. 55]):

Theorem A (Vitter [6]). Let $f: \boldsymbol{C}^{n} \rightarrow \boldsymbol{P}^{m}(\boldsymbol{C})$ be a meromorphic mapping of rank $n(m \geqq n+1)$ and

$$
F \equiv f \wedge f_{z_{1}} \wedge f_{z_{2}} \wedge \cdots \wedge f_{z_{n}}: \boldsymbol{C}^{n} \longrightarrow G(n+1, m+1) \subset \boldsymbol{P}^{\left(\begin{array}{c}
m+1 \\
n+1
\end{array}\right)-1}(\boldsymbol{C})
$$

the tangent mapping of $f$. Then

$$
T_{F}(r) \leqq(n+1) T_{f}(r)+O\left(\log r \cdot T_{f}(r)\right) \quad / /,
$$

where $G(n+1, m+1)$ denotes the Grassmanian manifold, $T_{g}(r)$ the Nevanlinna's characteristic function of a meromorphic mapping $g$ into a projective space and the notation "//" means that the stated inequality holds outside exceptional intervals.

In this note, we give an application of Theorem $\mathrm{A}$ as follows :

Let $V$ be a smooth hypersurface of degree $d$ in the projective space $\boldsymbol{P}^{n+1}(\boldsymbol{C})$. Then if $d>n+2$, any holomorphic mapping $f: C^{n} \rightarrow V$ must be degenerate in the sense that its Jacobian determinant vanishes identically.

Green [3] showed this theorem for $n=1$. (See also Carlson-Griffiths [1] and Kodaira [5].

We give another proof than that of Carlson-Griffiths and Kodaira, but they showed results under more general situations, e.g., $V$ is an $n$-dimensional projective algebraic manifold of general type.

Received March 9, 1983 


\section{Notation and Terminology.}

Let $z=\left(z_{1}, \cdots, z_{n}\right) \in \boldsymbol{C}^{n}$ be the natural coordinate system in $\boldsymbol{C}^{n}$. We set $\|z\|^{2}=\sum_{j=1}^{n} z_{j} \bar{z}_{j}, \quad \phi=d d^{c} \log \|z\|^{2}, \quad \phi_{k}=\phi \wedge \cdots \wedge \phi\left(k\right.$-times), $\quad \sigma=d^{c} \log \|z\|^{2} \wedge \phi_{n-1}, \quad B(r)$ $=\left\{z \in \boldsymbol{C}^{n} \mid\|z\|<r\right\}, \partial B(r)=\left\{z \in \boldsymbol{C}^{n} \mid\|z\|=r\right\}$ and $d^{c}=\frac{\sqrt{-1}}{4 \pi}(\bar{\partial}-\partial)$.

Let $L$ be a positive line bundle over $\boldsymbol{P}^{m}(\boldsymbol{C})$ and $\omega$ the curvature form for the line bundle $L$. For a holomorphic mapping $f: \boldsymbol{C}^{n} \rightarrow \boldsymbol{P}^{m}(\boldsymbol{C})$, we set

$$
T_{f}(L, r) \equiv \int_{0}^{r} \frac{d t}{t} \int_{B(t)} f^{*} \omega \wedge \phi_{n-1},
$$

where $f^{*} \omega$ denotes the pullback of the form $\omega$ under $f$. The function $T_{f}(L, r)$ is called the Nevanlinna's characteristic function of $f$ relative to $L$. In particular, for the hyperplane bundle $[H]$ over $\boldsymbol{P}^{m}(\boldsymbol{C})$ and the curvature form $\omega_{0}=$ $d d^{c} \log \|w\|^{2}\left(w \in C^{m+1}-\{0\}\right)$ of the canonical hermitian metric in $[H]$, we use the notation $T_{f}(r)$ instead of $T_{f}([H], r)$ for simplicity.

We note that for a holomorphic mapping $f: \boldsymbol{C}^{n} \rightarrow V \hookrightarrow_{\rightarrow} \boldsymbol{P}^{m}(\boldsymbol{C})$ with reduced representation $f=\left(f_{0}, \cdots, f_{m}\right)$, its characteristic function $T_{f}(r)$ is written in the form

$$
T_{f}(r)=\int_{\partial B(r)} \log \sum_{j=0}^{m}\left|f_{\jmath}\right|^{2} \sigma+O(1)
$$

\section{Applying Vitter's theorem, we show the following.}

THEOREM. Let $V \subset \boldsymbol{P}^{n+1}(\boldsymbol{C})$ be an n-dimensional smooth hypersurface given by a homogeneous polynomial $P(w)$ of degree $d$ and $d P(w) \neq 0$ in $w \in C^{n+2}-\{0\}$, and $f: \boldsymbol{C}^{n} \rightarrow V$ a holomorphic mapping. Then if $d>n+2, f$ must be degenerate in sense that its Jacobian determiant $J_{f}$ vanishes identically.

Proof. By definition of $V, P(f(z))=0$ on $C^{n}$. Hence differentiating this equation in $z_{j}(j=1, \cdots, n)$, we have

$$
\sum_{i=0}^{n+1} P_{i} \cdot f_{\imath}(z)_{z_{j}}=0 \quad(j=1, \cdots, n)
$$

where $P_{\imath}=\frac{d P}{d w_{i}}$. On the other hand, we have by Euler's formula

$$
\sum_{i=0}^{n+1} w_{i} P_{i}=0 \quad \text { on } \quad V
$$

so that

$$
\sum_{i=0}^{n+1} f_{\imath}(z) \cdot P_{i}(f(z))=0
$$

Suppose that $J_{f} \not \equiv 0$. Then there is at most one $j$ with $\Delta_{j} \not \equiv 0$, where 


$$
\Delta_{j}=\left|\begin{array}{c}
(\hat{j}) \\
f_{0} \cdots \cdots f_{j} \cdots \cdots f_{n+1} \\
\left(f_{0}\right)_{z_{1}} \cdots\left(f_{j}\right)_{z_{1}} \cdots\left(f_{n+1}\right)_{z_{1}} \\
\cdots \cdots \cdots \cdots \cdots \cdots \cdots \cdots \cdots \cdots \cdots \\
\left(f_{0}\right)_{z_{n}} \cdots\left(f_{j}\right)_{z_{n}} \cdots\left(f_{n+1}\right)_{z_{n}}
\end{array}\right| .
$$

Solving the equations (1) and (2) in $P_{i}(f(z))(i=0,1, \cdots, n+1)$, we have the ratio of $P_{0}: \cdots: P_{n+1}$;

$$
\left(P_{0}: \cdots: P_{n+1}\right)=\left(\frac{\Delta_{0 j}}{\Delta_{j}} P_{\jmath}: \cdots: \frac{\Delta_{n+1} j}{\Delta_{j}} P_{j}\right)
$$

for $j$ with $\Delta_{j} \neq 0$, where

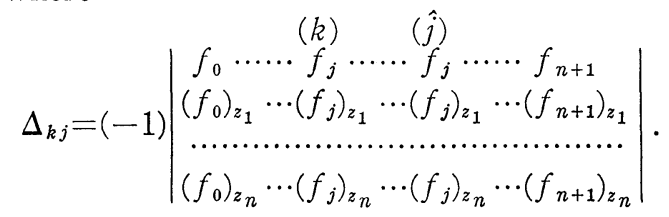

Here " $\wedge$ " over $(j)$ means that this column vector is to be deleted. We now denote by $G$ the left hand side and by $\Phi$ the right hand side of (3). Since the functions $P_{i}(i=0, \cdots, n+1)$ have not common zero, $h \equiv \sum_{i=0}^{n+1}\left|P_{i}\right|^{2}$ can be considered as a metric in $\left.[H]^{d-1}\right|_{V}$ which is the restriction over $V$ of the $(d-1)$-th symmetric tensor power $[H]^{d-1}$ of the hyperplane bundle $[H]$ over $\boldsymbol{P}^{n+1}(\boldsymbol{C})$. On the other hand, $\tilde{h} \equiv \sum_{i=0}^{n+1}\left|w_{\imath}^{d-1}\right|^{2}$ is also a metric in $\left.[H]^{d-1}\right|_{V}$. Thus we have

$$
\begin{aligned}
T_{G(f)}(r) & =\int_{\partial B(r)} \log \sum_{i=0}^{n+1}\left|P_{i}(f)\right|^{2} \sigma+O(1) \\
& =T_{f}\left(\left.[H]^{d-1}\right|_{V}, r\right)+O(1) \\
& =\int_{\partial B(r)} \log \sum_{i=0}^{n+1}\left|f_{i}^{d-1}\right|^{2}+O(1) \\
& =\int_{\partial B(r)} \max _{i} \log \left|f_{i}^{d-1}\right|^{2}+O(1) \\
& =(d-1) \cdot T_{f}(r)+O(1) .
\end{aligned}
$$

By definition we have

$$
\Phi \equiv\left(\Delta_{0 j} \frac{P_{j}}{\Delta_{j}}: \cdots: \Delta_{n+1} j \frac{P_{j}}{\Delta_{j}}\right)=\left(\Delta_{0 j}: \cdots: \Delta_{n+1} j\right)
$$

on $C^{n}-E$, where $E=\left\{z \in C^{n} \mid \Delta_{j}(z)=0\right\}$. Let $\delta$ be the common factor of $\Delta_{0 j}, \cdots, \Delta_{n+1 j}$ such that $F=\left(\frac{\Delta_{0 j}}{\delta}: \cdots: \frac{\Delta_{n+1 j}}{\delta}\right)$ is a reduced representation of $\Phi$. Note that $F$ is the tangent mapping 


$$
F=f \wedge f_{z_{1}} \wedge \cdots \wedge f_{z_{n}}: \boldsymbol{C}^{n} \longrightarrow G(n+1, n+2) \subset \boldsymbol{P}^{\left(\begin{array}{l}
n+2 \\
n+1
\end{array}\right)-1}(\boldsymbol{C})=\boldsymbol{P}^{n+1}(\boldsymbol{C}) .
$$

Hence we have by Theorem A

$$
\begin{aligned}
T_{G(f)}(r) & =T_{F}(r)=(d-1) T_{f}(r)+O(1) \\
& \leqq(n+1) T_{f}(r)+O\left(\log r \cdot T_{f}(r)\right) \quad / /
\end{aligned}
$$

Thus if $f$ is not rational and if $d>n+2$, the inequality (4) yields a contradiction for sufficiently large values of $r$. Thus we see $\Delta_{j} \equiv 0$ for all $j$. Therefore the Jacobian determinant of $f$ vanishes identically.

Suppose that $f$ is a rational mapping. Then $f$ can be written in the form $f=\left(1, Q_{1} / R_{1}, \cdots, Q_{n+1} / R_{n+1}\right)$ where $Q_{j}$ and $R_{j}$ are polynomials. We now consider a generic complex line $\boldsymbol{C}_{\xi}$ in $\boldsymbol{C}^{n}$, where $\xi$ is a direction vector in $\boldsymbol{C}^{n}$ and also we write a parameter in $\boldsymbol{C}_{\xi}$ as $\xi$ for simplicity. Then the restriction $\left.f\right|_{c_{\xi}}$ of $f$ to $C_{\xi}$ can be written in the form $f=\left(q_{0}(\xi): \cdots: q_{n+1}(\xi)\right)$, where $q_{0}, \cdots, q_{n+1}$ are polynomials in $\xi$ without common zero. Let $k_{i}$ be the degree of the polynomial $q_{i}$ and $k$ the maximum number among $k_{j}$ 's. Then we have

$$
\begin{aligned}
T_{\hat{f}}(r) & =\frac{1}{4 \pi} \int_{0}^{2 \pi} \log \sum_{i=0}^{n+1}\left|q_{i}\left(r e^{i \theta}\right)\right|^{2} d \theta+O(1) \\
& =k \cdot \log r+O(1)
\end{aligned}
$$

and hence

$$
T_{\hat{G}(f)}(r)=(d-1) \cdot T_{\hat{f}}(r)+O(1)=(d-1) k \cdot T_{\hat{f}}(r)+O(1),
$$

where $\hat{g}$ denotes the restriction of the function $g$ to $C_{\hat{s}}$. On the other hand from the right hand side of (3), we obtain

$$
\begin{aligned}
T_{\hat{F}}(r) & =\frac{1}{4 \pi} \int_{0}^{2 \pi} \log \sum_{n=0}^{n+1}\left|\widehat{\Delta}_{h j}\left(r e^{i \theta}\right)\right|^{2} d \theta-N\left((\hat{o})_{0}, r\right)+O(1) \\
& \leqq \frac{1}{4 \pi} \int_{0}^{2 \pi} \log \sum_{n=0}^{n+1}\left|\sum_{\left(\alpha_{i}\right)} \prod_{\substack{i=0 \\
i \neq k}}^{n+1} \hat{f}_{i}\left(r e^{i \theta}\right)_{z_{\alpha_{i}}}\right|^{2} d \theta+O(1) \\
& \leqq\{k(n+1)-n\} \log r+O(1),
\end{aligned}
$$

for the degree of $\prod_{\substack{i=0 \\ i \neq k}}^{n+1} \hat{f}_{i}(z)_{z_{\alpha_{i}}}$ is not greater than $k(n+1)-n$. Thus we have

$$
(d-1) k \cdot \log r+O(1) \leqq\{k(n+1)-n\} \cdot \log r+O(1),
$$

and hence

$$
\{k(d-n-2)+n\} \cdot \log r \leqq O(1) .
$$

Hence this gives a contradiction if $d \geqq n+2$. Thus we have $\Delta_{\jmath} \equiv 0$ for all $j$. Therefore $f$ is degenerate in the sense that $J_{f} \equiv 0$. 


\section{REFERENCES}

[1] J. Carlson and P. A. Griffiths, A defect relation for equidimensional holomorphic mappings between algebraic varieties, Ann. of Math., 95 (1972), 557-584.

[2] H. CARTAN, Sur les zéros des combinaisons linéares de $p$ fonctions holomorphes données, Mathematica, 7 (1933), 5-31.

[3] M. L. Green, Some Picard theorems for holomorphic maps to algebraic varieties, Amer. J. Math., 97 (1975), 43-75.

[4] W. K. Hayman, Meromorphic Functions, Oxford Math. Monographs, Oxford University Press, London (1964).

[5] K. Kodaira, Holomorphic mappings of polydiscs into compact complex manifolds, J. Differential Geometry, 6 (1971), 33-46.

[6] A. VitTer, The lemma of the logarithmic derivative in several complex variables, Duke Math. J., 40 (1977), 89-104.

Department of Mathematics

YAMAGATA UNIVERSITY

YAMAGATA, JAPAN 